\title{
Revisiting Relaxation Model towards Prediction of Longterm Mechanical Behavior of Semicrystalline Fibers ${ }^{\star}$
}

\author{
Christina Schippers ${ }^{a, *}$, Thomas Bahners ${ }^{a}$, Larisa A. Tsarkova ${ }^{a, b}$, Jochen S. Gutmann $^{a, c}$ and \\ Ernst Cleve ${ }^{d, *}$
}

${ }^{a}$ German Textile Research Center (DTNW), Adlerstraße 1, 47798 Krefeld, Germany

${ }^{b}$ Department of Chemistry, Moscow State University, 119991 Moscow, Russia

${ }^{c}$ Physical Chemistry, University Duisburg-Essen and CENIDE, Essen, Germany

${ }^{d}$ University of Applied Sciences, Krefeld, Germany

\section{ARTICLE INFO}

\section{Keywords:}

Maxwell model

Relaxation

Tensile test

Lifetime prediction

\begin{abstract}
A B S T R ACT
Tensile testing is a well-established method to assess the maximum strength of a material, while relaxation tests are used to evaluate the viscoelastic behaviour of a polymer. Because of slow viscoelastic changes, significant measurement times are required for reliable descriptions. Therefore the relaxation tests are usually combined with lifetime prediction models to reduce the experimental load. Various traditional models use the time-temperature superposition principle while modificated relaxation models are e.g. based on the time-strain superposition principle (TSSP). Both variations require several measurement series to set up a relaxation master curve (RMC). The basic assumption is that a higher strain corresponds to a higher temperature and a longer load duration, respectively. The paper describes a new model approach which allows to predict the longterm behaviour by using a reduced number of measurements as compared to widely models. The new model is based on the well-known Maxwell model and assumes a mean relaxation time in combination with a relaxation coefficient. These parameters account for the inhomogeneity of the individual polymer chains. A dimensionless number, similar to the relaxation coefficient, has been successfully introduced for the Weibull distribution and the particle size distribution. The new model allows to derive master curve from one measurement series at a single strain by fitting the data to the model equation.
\end{abstract}

\section{Introduction}

Material science includes two main scopes: design of new materials and the analysis of their properties, also in a view of the practical usage in targeted applications. Understanding the properties of novel polymer materials, including rapidly growing class of bio-based polymers, implies a detailed characterisation of their mechanical and thermal behaviour, particularly under application conditions. A great challenge in transferring the scientific knowledge into new materials and new technologies is the assessment of a lifetime and durability on a time scale of months or even years. In praxis, lifetime prediction approaches are preferred over realtime measurements, which are in most instances not feasible to perform. Especially viscoelastic polymers are known to exhibit time-dependent mechanical properties [9] which are even more pronounced for newly developing biobased and bio-degradable polymers[7].

Tensile testing is a well-established method to assess the maximum strength of a polymer material, while relaxation tests are used to evaluate their viscoelastic behaviour. Due

\footnotetext{
${ }^{\star}$ The ongoing research project (IGF-Nr. 19812 BG) of the „Forschungskuratorium Textil e.V. (FKT)“ is funded by the „Arbeitsgemeinschaft industrieller Forschungsvereinigungen e.V. (AiF)" within the program „Industrielle Gemeinschaftsforschung und -entwicklung (IGF)“ by the Federal Ministry for Economic Affairs and Energy on the basis of a decision by the German Bundestag.

* Corresponding author

(1) schippers@dtnw.de (C. Schippers)

e www.dtnw.de (C. Schippers)

$\operatorname{ORCID}(\mathrm{s})$ :
}

to the slow viscoelastic changes, a prolonged measurement time is required for reliable descriptions. Therefore, the relaxation tests are usually combined with lifetime prediction models to reduce the experimental load. Traditional relaxation models are based on time-temperature superposition principle (TTSP), and require several measurement series to set up a relaxation master curve (RMC). The basic assumption is that a higher temperature corresponds to a longer load duration.

The first attempts to quantify mechanical properties date back two centuries. At that time, model equations were applied to describe correlations in experimentally measured behaviour. The handling of the measured data can be standardised by application of a universal evaluation, thus allowing to reduce the measuring efforts and time.[3] A wellknown example of a mechanical model was developed by Maxwell (Maxwell model [10] [3]).

Already in 1868 Maxwell described the elastic deformation with a spring (Hooke's law) and the viscoelastic deformation with a dashpot (Newton's law). By using a linear arrangement, the stress-strain behaviour as well as the relaxation behaviour can be modulated. To discuss the creep deformation, Voigt proposed a linear arrangement of the spring and the dashpot in 1928. This arrangement leads to a modulation of the creep behaviour while missing the relaxation behaviour. [1] For a more realistic description it is recommended to connect several Maxwell or Voigt elements in series, which is then called generalized Maxwell model and generalized Voigt model, respectively. [3]

While the importance of mechanical models increased, 
several scientists proposed their own ideas to modulate the mechanical properties. In 1945, Alfrey and Doty [1] have made the afford to summarize and compare all existing methods, describing the properties of incompressible, isotropic, viscoelastic materials. They defined two classes. The first one contains the fundamental models like proposed by Maxwell and Voigt, while the second class is defined by model equations evaluated from valid measurement data. [1] proved that all described models they are mathematical equivalent and proposed to combine the methods to get full information about the longterm behaviour. For this purpose, the measurement data can be used as a basis for small measurement times, while the continuation of the model curve is done with the help of a class 1 model (Maxwell/Voigt model).

An alternative approach to continue the model curve, based on measurements data, is the use of the time-temperature superposition principle (TTSP), also known as Williams-LandelFerry (WLF) method. [13] [6] Leaderman [8] observed, that the time is equivalent to the temperature. [5] This correlation allows to predict the longterm behaviour while using an empirical time shift on a $\log t$ axis. This results in measurements, where the temperature instead of the time is increased to significantly reduce the measurement effort. By using a set of shortterm measurements at different temperatures it is possible to calculate a specific time shift for each temperature to form a relaxation master curve $(R M C)$ which predicts the longterm behaviour over several time decades. [13] [9]

Today are several more methods published to predict the behaviour of viscoelastic materials, than described by Alfrey and Doty in 1945. One aspect, they all have in common, is that they are based on one of the previous considerations. The most common one is the time-temperature superposition principle (TTSP) or modificated time superposition principles. [9] The TTSP works well for amorphous polymers but misses the sufficient description of semi-crystalline or crystalline polymers. That is why, in most cases, polymethyl methacrylate (PMMA), a typical amorphous polymer, is used to present the results of the TTSP. [13] Another limitation of the TTSP is the temperature range. The behaviour in the given temperature range needs to follow the linear Arrhenius relationship [4], therefore only temperatures below the glass transition temperature $T_{g}$ can be used. The range for using a modification of the time superposition principle e.g. the time-stress superposition principle (TSSP) is similar, the stress needs to be linear, like given in the linear elastic area, defined by tensile tests.

This paper describes a new approach to define a model equation, based on the considerations of Maxwell [10] from 1868, which allows to predict the longterm behaviour. While using a reduced number of measurements, compared to the well known time-temperature superposition principles, the use of the new model equation is very effective. The theoretical considerations are supported by a set of simulation data. The evaluation is carried out by comparing the new model with an example of the TSSP (Schulz model [12]).

\subsection{Schulz model for material lifetime prediction}

The Schulz model represents the various methods of lifetime prediction using the time superposition principle. Schulz et al. [12] used a approach by Persoz [11] to define a model equation (see equation 1 ). This equation allows to define a relaxation master curve $R M C$ predicting the relaxation behaviour over a longer time period while performing shortterm measurements.

$$
\sigma(t)=E_{0} \cdot \varepsilon-\left(E_{0}-E_{\infty}\right) \cdot \varepsilon \cdot \Phi_{\varepsilon}(t)
$$

The stress $\sigma$, as a function of the time $t$, is outlined by the initial modulus $E_{0}$ and the final modulus $E_{\infty}$ with a constant elongation $\varepsilon$. The impact of $t$ is only given by the normalized relaxation function $\Phi_{\varepsilon}(t)$.

In 2013, Bahners et al. [2] demonstrated how to evaluate the data of short-term relaxation measurements for a semi- crystalline poly(ethylene terephthalate) (PET) fibre according to the Schulz model. Since the modulus $E$ of a fibre, with constant elongation $\varepsilon$, follows a symmetrical s-shaped curve if plotted against the logarithm of time [12], the normalized relaxation function $\Phi(t)$ can be described by the integral over the Gauss normal distribution as shown in equation 2.

$$
\Phi(t)=\frac{1}{\sqrt{2 \pi}} \cdot \int_{-\infty}^{V_{\varepsilon}(t)} \exp \left(\frac{-z^{2}}{2}\right) d z
$$

The integration limit $V_{\varepsilon}(t)$, defined by equation 3 , is a function of the running time $t$. The logarithm of the relaxation time $\tau_{\varepsilon}$ marks the symmetry point. For the scaling of the curve the shape factor $a$ is used.

$$
V_{\varepsilon}(t)=\frac{1}{a}\left(\log t-\log \tau_{\varepsilon}\right)
$$

By combining the three previous equations 1 to 3 it appears that the longterm behaviour of polymers can be described by using four parameters: $E_{0}, E_{\infty}, a$ and $\tau_{\varepsilon}$. Like suggested by [1], the original measurements data should be used to described the beginning of the $R M C$. So the initial modulus $E_{0}$ can easily be found by using the original measurement data at time $t=0$. The other parameters must be found by evaluating a set of short-term relaxation measurements with variable elongations $\varepsilon$. According to the time superpositions the higher $\varepsilon$ represents the ageing of the polymer. This time superposition is implicated by an elongation-dependent timeshift $\Delta t_{\varepsilon}$ from experimental time $t$ to time $t^{\prime}$ along the $\log t$ axis as shown in equation 4.

$$
\log t^{\prime}=\log t+\log \Delta t_{\varepsilon}
$$

According to the symmetrical s-shaped curve, like assumed for the Schulz model, the plot of the derivation of the modulus $E^{\prime}$ against the modulus $E$ needs to have a parabolic shape. The minimum of this curve marks the symmetry point of the RCM at relaxation time $\tau_{\varepsilon}$, defined as vertex $\left(E_{\tau} / E_{\tau}^{\prime}\right)$.

With a given value for the symmetry point $E_{\tau}$, which mathematically defines the midway between the initial modulus $E_{0}$ and the final modulus $E_{\infty}$, the missing value for the final modulus $E_{\infty}$ can be calculated by using equation 5 .

$$
E_{\infty}=E_{0}-2 E_{\tau}
$$


Additionally, the symmetry point $E_{\tau}$ can be used to calculate the integration limit $V_{\varepsilon}(t)$ (equation 6) and the shape factor $a$ (equation 7).

$$
\begin{aligned}
& V_{\varepsilon}\left(t_{1}^{\prime}\right)=\sqrt{2 \cdot \ln \left(\frac{E_{\tau}^{\prime}}{E^{\prime}\left(t_{1}\right)}\right)} \\
& a=-\frac{\Delta E}{\sqrt{2 \pi} \cdot E_{\tau}^{\prime}}
\end{aligned}
$$

The last parameter, to define the whole $R M C$, is the logarithmic time shift $\tau_{\varepsilon}$, which can be calculated by using equation 3. Thus, a series of short-term measurements can be used to calculate all parameters that are necessary to set up the complete $R M C$ of the Schulz model, with the elastic modulus $E(t)$ depending on the time $t$, as presented in equation 8 .

$$
E(t)=E_{0}-\left(E_{0}-E_{\infty}\right) \cdot \frac{1}{\sqrt{2 \pi}} \cdot \int_{-\infty}^{V_{\varepsilon}(t)} \exp \left(\frac{z^{2}}{2}\right) d z
$$

Based on the previous equations, Bahners et al. [2] used some recalculation of all given parameters to approximate the $R M C$ to the measurement data. The complete evaluation process would go beyond the scope of this article and can be found in the corresponding article from Bahners et al. [2].

\section{Concept of new relaxation model}

The traditional models for material lifetime prediction, based on time superpositions, require a high measurement effort. As described above, there are several shortterm measurements needed to define the longterm model equation for the $R M C$.

The new relaxation model, which also describes the longterm behaviour of a viscoelastic polymer is based on the generalized Maxwell model [10]. Without the use of the time superpositions, there are less measurements needed to set up the model curve. The related parameters can be evaluated by using only one, instead of several measurement series to define the model equation.

The considerations of Maxwell start with a linear spring and a linear viscous dashpot like presented in equations 9 10. While the spring describes the elastic behaviour, following Hook's law, the dashpot describes the time-dependent viscous behaviour, influenced by the strain rate $\dot{\varepsilon}_{d}$ and the viscosity $\eta$. [1]

$$
\begin{aligned}
& \sigma_{s}=E \cdot \varepsilon_{s} \\
& \sigma_{d}=\eta \cdot \dot{\varepsilon_{d}}
\end{aligned}
$$

With a linear arrangement of these two elements the resulting strain $\varepsilon$ of the Maxwell element is a combination of the single strains for the spring $\varepsilon_{s}$ and the dashpot $\varepsilon_{d}$ while the stress $\sigma$ is constant in every element.

$$
\varepsilon=\varepsilon_{s}+\varepsilon_{d}
$$

$$
\sigma=\sigma_{s}=\sigma_{d}
$$

The transformation of equations 9 - 11 allows to define the equation for the Maxwell model.

$$
\dot{\varepsilon}=\frac{\dot{\sigma}}{E}+\frac{\sigma}{\eta}
$$

In case of relaxation measurements the strain $\varepsilon$ is constant and the strain rate $\dot{\varepsilon}$ is zero. With these conditions, Maxwell's differential equation 12 can be solved to obtain the Maxwell model relaxation equation 14 with the relaxation time $\lambda$ being defined by:

$$
\begin{gathered}
\lambda=\frac{\eta}{E} \\
0=\frac{1}{E} \frac{d \sigma}{d t}+\frac{\sigma}{\eta} \\
\frac{d \sigma}{\sigma}=-\frac{E}{\eta} \cdot d t \\
\sigma(t)=\sigma_{0} \cdot \exp \left(-\frac{t}{\lambda}\right)
\end{gathered}
$$

A combination of more than two elements, also known as generalized Maxwell model, allows a more detailed description of the mechanical behaviour. The parallel arrangement of $N$ Maxwell elements and a single spring can be expressed as addition of the stresses for the Maxwell elements $\sigma_{i}$ and the stress $\sigma_{\infty}$. The evaluation as modulus $E$ instead of stress $\sigma$ makes the data independent of the given strain $\varepsilon$.

$$
\begin{gathered}
\sigma(t)=\sigma_{1} \cdot \exp \left(-\frac{t}{\lambda_{1}}\right)+\sigma_{2} \cdot \exp \left(-\frac{t}{\lambda_{2}}\right)+\ldots+\sigma_{\infty} \\
\sigma(t)=\sigma_{\infty}+\sum_{i=1}^{N} \sigma_{i} \cdot \exp \left(-\frac{t}{\lambda_{i}}\right) \\
E(t)=E_{\infty}+\sum_{i=1}^{N} E_{i} \cdot \exp \left(-\frac{t}{\lambda_{i}}\right)
\end{gathered}
$$

With these foundations, the considerations for the new relaxation model are applied.

1. The relaxation time coefficient $\lambda_{i}$ is a material constant. A mean relaxation time coefficient $\bar{\lambda}$ is defined

$$
E(t)=E_{\infty}+\exp \left(-\frac{t}{\bar{\lambda}}\right) \cdot \sum_{i=1}^{N} E_{i}
$$

2. A relaxation coefficient $n$ is applied, which takes the inhomogeneity of the individual polymer chains into account. A similar dimensionless number has also been successfully introduced for the Weibull distribution and the particle size distribution according to the RRSB model.

$$
E(t)=E_{\infty}+\exp \left(-\left(\frac{t}{\bar{\lambda}}\right)^{n}\right) \cdot \sum_{i=1}^{N} E_{i}
$$


3. The initial modulus $E_{0}$ is defined by the modulus at time $t=0$, which results in the sum of the individual moduli $E_{i}$ being expressed by the difference of the initial and final modulus.

$$
\begin{gathered}
E(t=0)=E_{0}=E_{\infty}+\exp \left(-\left(\frac{0}{\bar{\lambda}}\right)^{n}\right) \cdot \sum_{i=1}^{N} E_{i} \\
\sum_{i=1}^{N} E_{i}=E_{0}-E_{\infty}
\end{gathered}
$$

The overall model equation for the new relaxation model is presented as equation 19 .

$$
E(t)=E_{\infty}+\left(E_{0}-E_{\infty}\right) \cdot \exp \left(-\left(\frac{t}{\bar{\lambda}}\right)^{n}\right)
$$

With the moduli $E_{0}, E(t)$ as well as time $t$ being extracted from the original measurement data, the missing parameters $E_{\infty}, \lambda$ and $n$ can be calculated by using the method of least squares.

\section{Set up simulation data}

For the evaluation of both previously described model (Schulz model according to Bahners et al. and new relaxation model) some simulation data are generated. For a successful simulation, some scientific laws must be followed.

\section{Hooke's law}

The initial modulus $E_{0}$ is constant within the elastic range.

\section{Generalized Maxwell model}

The final modulus $E_{\infty}$ is constant.

\section{Newton's law}

With increasing strain $\varepsilon_{i}$, the relaxation time coefficient $\lambda_{i}$ decreases with linear dependence.

Due to these laws, the following simulations parameters in Table 1 are set up. In compliance with equation 19, the fol-

Table 1

Simulation parameters for evaluating the relaxation models.

\begin{tabular}{ccccc}
\hline$\varepsilon_{i}(\%)$ & $\lambda_{i}(s)$ & $E_{0}(M P a)$ & $E_{\infty}(M P a)$ & $n$ \\
\hline 1,0 & 600 & & & \\
2,0 & 540 & & & \\
3,0 & 480 & 5000 & 1000 & 1,00 \\
4,0 & 420 & & & \\
5,0 & 360 & & & \\
6,0 & 300 & & & \\
\hline
\end{tabular}

lowing curves in Figure 1 a-c can be determined. As already presented in the previous chapter, plotting on a logarithmic time axis is preferred for further evaluation. The curves of the simulation data clearly show the typical, symmetrical sshaped curv often described in literature [1]
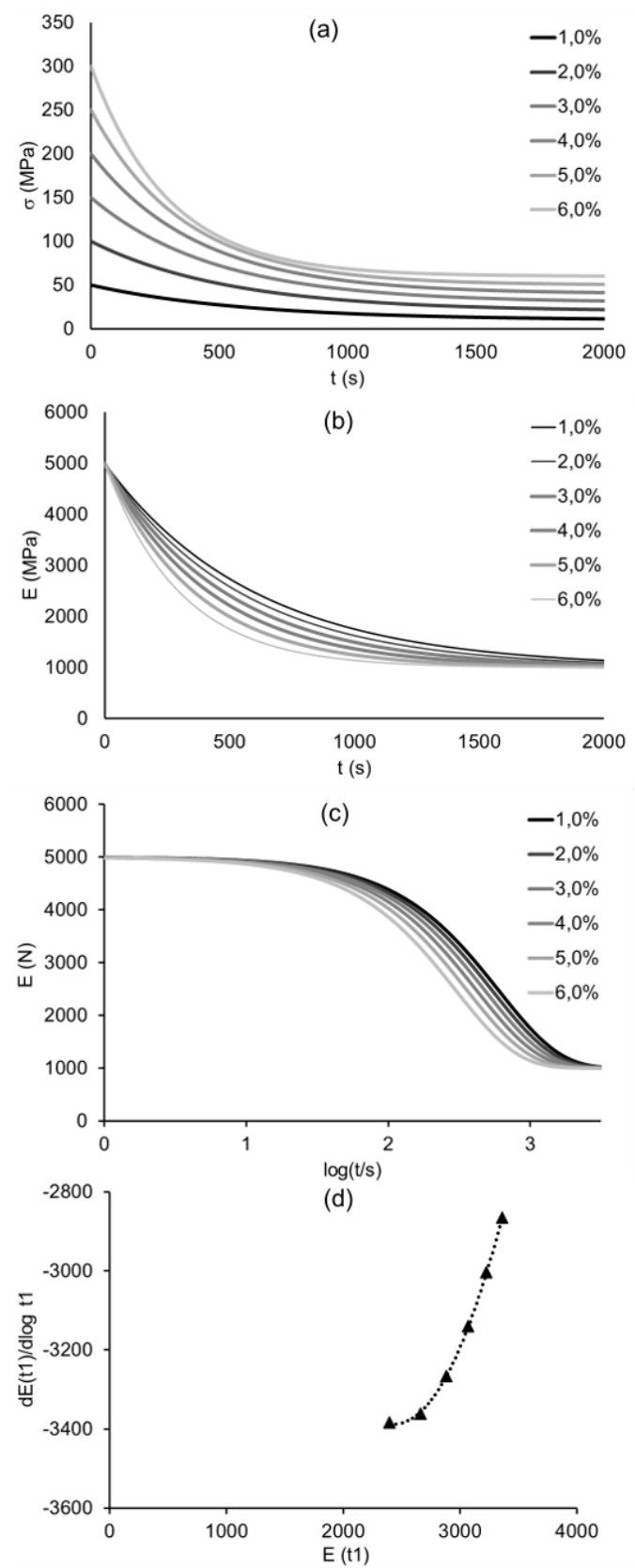

Figure 1: Set of simulation data for evaluating the Schulz model.

\section{Evaluation of new relaxation model}

The evaluation of the new relaxation model requires considerably less effort than the evaluation of a model based on a time superposition principle. With the given model equation 19 and the related deviation the parameters can easily be extracted from the data.

$$
E(t)=E_{\infty}+\left(E_{0}-E_{\infty}\right) \cdot \exp \left(-\left(\frac{t}{\bar{\lambda}}\right)^{n}\right)
$$

As already described for the Schulz model, the plot of the derivation of the modulus against the modulus has a parabolic shape. The minimum marks the turning point of the modulus plotted against the logarithm of time (see figure $1 \mathrm{c}$ ). 
Therefore the analytical derivation of the model equation 19 according to $\log t$ is calculated by the previous derivation according to $t$.

$$
\begin{gathered}
\frac{d E(t)}{d t}=\left(E_{0}-E_{\infty}\right) \cdot \exp \left(-\left(\frac{t}{\bar{\lambda}}\right)^{n}\right) \cdot n \cdot\left(\frac{t}{\bar{\lambda}}\right)^{n-1} \cdot \frac{-1}{\bar{\lambda}} \\
d E(t)=\left(E_{0}-E_{\infty}\right) \cdot \exp \left(-\left(\frac{t}{\bar{\lambda}}\right)^{n}\right) \cdot n \cdot\left(\frac{t}{\bar{\lambda}}\right)^{n-1} \cdot \frac{-1}{\bar{\lambda}} \cdot d t \\
\frac{d \log t}{d t}=\frac{1}{t} \cdot \frac{1}{\ln 10} \\
d \log t=\left(\frac{1}{t} \cdot \frac{1}{\ln 10}\right) d t \\
\frac{d E(t)}{d \log t}=\left(E_{0}-E_{\infty}\right) \cdot \exp \left(-\left(\frac{t}{\bar{\lambda}}\right)^{n}\right) \cdot n \cdot\left(\frac{t}{\bar{\lambda}}\right)^{n-1} \cdot \frac{-1}{\bar{\lambda}} \cdot \ln 10 \cdot t
\end{gathered}
$$

The initial modulus is simply defined at the time $t=0$, while the remaining model parameters $E_{\infty}, \bar{\lambda}$ and $n$ require some mathematical conversion.

While plotting the derivation of the modulus against the modulus for all given strains $\varepsilon_{i}$ in figure $1 \mathrm{~d}$ it can be seen, that the six curves for the six strains perfectly overlap. Thereby the relaxation time $\bar{\lambda}$ is valid for the derivation of all strains $\varepsilon_{i}$ while the relaxation times $\lambda_{i}$ are related to the not derived modulus and individual for each strain.

Since all parameters of the model equation are positive, the minimum of the derivative function is given for $\bar{\lambda}=$ $t$. After graphical determination of the minimum $E_{\text {min }}=$ $\frac{d E(\bar{\lambda})}{d \log \bar{\lambda}}$, it is possible to use this value to calculate the final modulus $E_{\infty}$, like presented in eq. 27 . As well as the relaxation time $\bar{\lambda}$, the final modulus $E_{\infty}$ is not depending on the individual strains $\varepsilon_{i}$.

$$
E_{\text {min }}=\frac{d E(\bar{\lambda})}{d \log \bar{\lambda}}=\left(E_{0}-E_{\infty}\right) \cdot \exp \left(-\left(\frac{\bar{\lambda}}{\bar{\lambda}}\right)^{n}\right) \cdot n \cdot\left(\frac{\bar{\lambda}}{\bar{\lambda}}\right)^{n-1} \cdot \frac{-1}{\bar{\lambda}} \cdot \ln
$$

$$
E_{\text {min }}=\left(E_{0}-E_{\infty}\right) \cdot \exp (-1) \cdot n \cdot(-1) \cdot \ln 10
$$

$$
E_{\infty}=E_{0}-\frac{E_{\min }}{\exp (-1) \cdot n \cdot(-1) \cdot \ln 10}
$$

However, the evaluated minimum $E_{\text {min }}$ leads to the related modulus (see figure 1d), which defines the turning point of the s-shaped curve in figure $1 \mathrm{~b}$. This turning point is located at individual times $\mathrm{t}$ and defines the strain dependent relaxation time $\lambda_{i}$.
The last undefined parameter $n$ can be determined by inserted the given values for $E_{\text {min }}, E_{0}$ and $E_{\infty}$ in the transformed eq. 26 (see eq. 28). As the calculation does not use any values, which are depending on the given strain $\varepsilon_{i}, \mathrm{n}$ is valid for all given strains.

$$
n=\frac{E_{\min }}{\left(E_{0}-E_{\infty}\right) \cdot \exp (-1) \cdot(-1) \cdot \ln 10}
$$

The evaluated model parameters for the new relaxation model are presented in table 2 .

Table 2

Evaluated model parameters according to the new relaxation model.

\begin{tabular}{ccccc}
\hline$\varepsilon_{i}(\%)$ & $\lambda_{i}(s)$ & $E_{0}(M P a)$ & $E_{\infty}(M P a)$ & $n$ \\
\hline 1,0 & 603 & & 1000 & 1,00 \\
2,0 & 537 & & 1000 & 1,00 \\
3,0 & 479 & 5000 & 1000 & 1,00 \\
4,0 & 417 & & 1000 & 1,00 \\
5,0 & 363 & & 1000 & 1,00 \\
6,0 & 302 & & 1000 & 1,00 \\
\hline
\end{tabular}

\section{Evaluation of Schulz model}

The whole evaluation of the Schulz model is based on the symmetry points of the s-shaped curves, resulting from the plotting of the modulus $E$ against the logarithm of time $\log (t / s)$ like presented in figure $1 \mathrm{c}$. These symmetry points can easily be found by calculating the derivation of the modulus.

Due to the missing elementary function for the Gauss normal distribution, the derivation is calculated numerically by using the central derivation in eqn. 29.

$$
\frac{d E\left(t_{i}\right)}{d \log \left(t_{i} / s\right)}=\frac{E\left(t_{i+1}\right)-E\left(t_{i-1}\right)}{\log \left(t_{i+1} / s\right)-\log \left(t_{i-1} / s\right)}
$$

Therefore a random evaluation time is chosen. Plotting the deviation of the modulus against the modulus for all six strains at time $t_{1}$ results in a parabola with a minimum $\left(E_{\tau} / E_{\tau}^{\prime}\right)$ representing the symmetry points of the s-shaped curves in figure $1 \mathrm{c}$ at time $\tau$. To get this parabola, the symmetry point at any of the six curves of the simulation data need to be covered. For

$$
\log \left(\frac{t_{1}}{1 s}\right)=2,5
$$

the evaluation time is close to the symmetry point of the curve for $\varepsilon_{i}=6 \%$, while an increasing time shifts the related symmetry point to a lower strain. When the chosen time is not in the area of any of the symmetry points, the plot of the deviation of the modulus against the modulus does not form a parabola and the minimum can not be evaluated correctly.

By increasing the random evaluation time, it points out that the model curve can be approximated to the curve of the simulation data. At

$$
\log \left(\frac{t_{1}}{1 s}\right)=2,7
$$




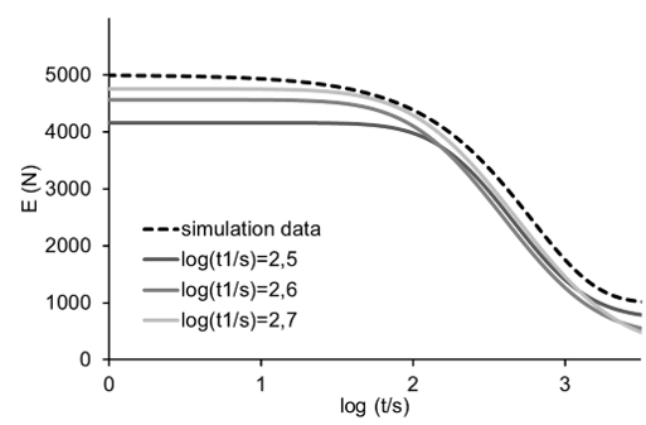

Figure 2: Evaluated model curves (gray) with varying evaluation times in comparison to simulation data with $\varepsilon=1 \%$ (dashed black).

the maximum time is reached while covering the symmetry point of the curve for $\varepsilon_{i}=1 \%$.

Whith the evaluated values for $E_{\tau}, E_{\tau}^{\prime}$ and $\log \tau$ all missing parameters to set up the Schulz model equation can be calculated by using equations 5 to 7 . The resulting model curves for the varying evaluation times are presented in figure 2 (gray) with the resulting parameters in table 3.

\section{Table 3}

Model parameters for evaluated simulation data in table 1 according to the Schulz model.

\begin{tabular}{ccccc}
\hline $\log \left(t_{1} / s\right)$ & $\log (\tau / s)$ & $E_{0}(M P a)$ & $E_{\infty}(M P a)$ & $a$ \\
\hline 2,5 & 2,7 & 4159 & 727 & 0,40 \\
2,6 & 2,6 & 4565 & 421 & 0,49 \\
2,7 & 2,7 & 4755 & 193 & 0,54 \\
\hline
\end{tabular}

Even with a optimized evaluation time, the overlapping of the simulation data and the evaluated model curve in figure 2 is not ideal. Especially the final modulus for the model curve $\left(E_{\infty}=193 \mathrm{MPa}\right)$ is far away from the value chosen for the simulation data $\left(E_{\infty}=1000 \mathrm{MPa}\right)$. This can easily explained by the evaluation of the minimum of the presented parabola in figure $1 \mathrm{~d}$, which defines, according to Schulz and the applied Gauss normal distribution, the middle between the final and the elastic modulus. In case of the new model, the s-shaped curve of the modulus against the logarithm of time is not perfectly symmetrical. The turning point of the curve is not defining the middle between the final and the elastic modulus. This can be shown by plotting the deviation of the modulus against the modulus at every evaluation time in figure 3 . This missing symmetry results in a shifted turning point which leads to wrong values for the initial and final modulus, even if the difference of the moduli is calculated correctly. However there is one case, where the fitting of the simulation data and the model curve can still be improved. This case is not changing the evaluation process but the simulation data. When the shaping factor $n$ is reduced, the entire curve becomes flatter. This increases the changes at the beginning and the end of the curve and minimizes the influce of the divergent initial and final modulus. This describes the Schulz model as valid method for

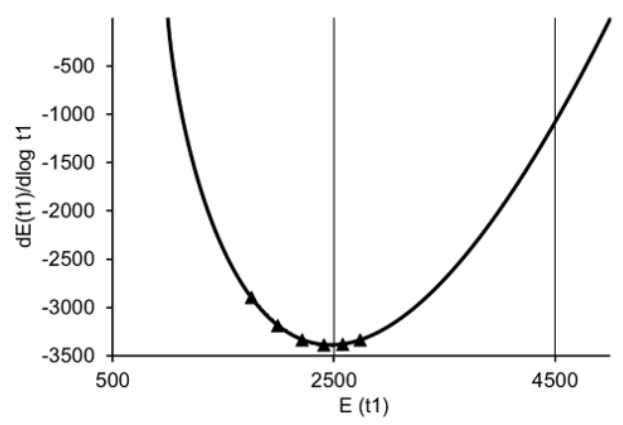

Figure 3: Deviation of modulus against modulus at varying evaluation times for simulation data with $\varepsilon=1 \%$. Solid triangles are representing the calculated values at evaluation time $\log \left(t_{1} / s\right)=2,7$ for all simulated strains.

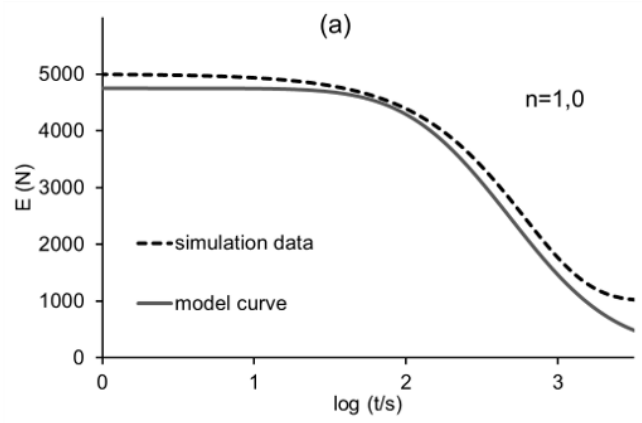

(b)

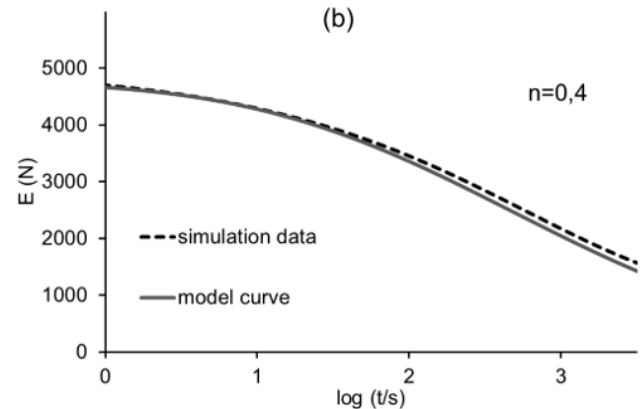

Figure 4: Evaluated model curves (gray) with varying shaping factors $n$ used to set up the simulation data evaluation: (a) $n=1$; (b) $n=0,4$ in comparison to simulation data with $\varepsilon=1 \%$ (dashed black).

lifetime prediction as long as the curve is flat enough to be represented by a symmetrical gauss normal distribution.

\section{Conclusion}

The design of a new relaxation model is an interesting field of research, as long as the model is an improvement compared to all previous assumptions. One aspect to be improved can be the validated material. The modelling of semi-crystalline materials is much more complicated than the modelling of amorphous materials. In this paper, the focus is not on the different materials, but on a reduction of the measuring effort. With the new relaxation model a master curve can be designed which does not differ significantly from the Schulz model, which deals as example for the evaluation via time-stress superposition principle (TSSP). The 
reduced measuring effort is characterized by the model curve which can be created by using one single measurement. In contrast to this is the Schulz Model, which requires several curves with defined time shifts for the composition of the model curve. Additionally, the accuracy of the model curve can be characterized by the inclusion of further measurement data.

\section{References}

[1] Alfrey, T., Doty, P., 1945. The methods of specifying the properties of viscoelastic materials. Journal of Applied Physics 16, 700-713. doi:10.1063/1.1707524.

[2] Bahners, T., Schmidt, M., Gutmann, J.S., 2013. Correlation of material lifetime predictions by artificial aging vs. the relaxation master curve. Polymer Bulletin 70, 1659-1676. doi:10.1007/ s00289-013-0951-y.

[3] Berry, J.P., Ferry, J.D., Jenckel, E., Kast, W., Mark, H.F., Meskat, W., Rosenberg, O., Schwarzl, F., Staverman, A.J., Stein, R.S., Stuart, H.A., Thurn, H., Treloar, L.R.G., Vegt, A.K., Würstlin, F., 1956. Theorie und molekulare Deutung technologischer Eigenschaften von hochpolymeren Werkstoffen. volume 4 of Die Physik der Hochpolymeren. Springer, Berlin and Heidelberg. URL: https: //books. google. de/books?id=BUjzBgAAQBAJ, doi:10.1007/978-3-662-01378-6.

[4] Celina, M., Gillen, K.T., Assink, R.A., 2005. Accelerated aging and lifetime prediction: Review of non-arrhenius behaviour due to two competing processes. Polymer Degradation and Stability 90, 395404. doi:10.1016/j. polymdegradstab. 2005.05.004.

[5] Cowie, J.M.G., 1991. Polymers: chemistry and physics of modern materials. 2. ed. ed., Blackie, Glasgow.

[6] Ferry, J.D., 1980. Viscoelastic properties of polymers. Third edition ed., John Wiley \& Sons, New York and Chichester and Brisbane and Toronto and Singapore.

[7] Laycock, B., Nikolić, M., Colwell, J.M., Gauthier, E., Halley, P., Bottle, S., George, G., 2017. Lifetime prediction of biodegradable polymers. Progress in Polymer Science 71, 144-189. doi:10.1016/j. progpolymsci.2017.02.004.

[8] Leaderman, H., 1943. Elastic and creep properties of filamentous materials and other high polymers. The Textile foundation.

[9] Luo, W., Wang, C., Hu, X., Yang, T., 2012. Long-term creep assessment of viscoelastic polymer by time-temperaturestress superposition. Acta Mechanica Solida Sinica 25, 571578. URL: http://www. sciencedirect.com/science/article/pii/ S0894916612600524, doi:10.1016/s0894-9166(12)60052-4.

[10] Maxwell, J.C., 1868. On the dynamical theory of gases. The London, Edinburgh, and Dublin Philosophical Magazine and Journal of Science 35 .

[11] Persoz, B., 1957. Le principle de superposition de boltzmann. Canier Groupe France, Etudes Rheology 2, 126-281.

[12] Schulz, J., Stalevitsch, A., Eickmeier, A., Bossmann, A., Schollmeyer, E., 1996. Modell zur beschreibung der viskoelastischen eigenschaften von synthetischen fasern. Die Angewandte Makromolekulare Chemie 234, 159-175. URL: https: //onlinelibrary.wiley.com/doi/pdf/10.1002/apmc.1996.052340114, doi: $10.1002 /$ apmc. 1996.052340114.

[13] Urzhumtsev, Y.S., 1976. Time-temperature superposition. review. Polymer Mechanics 11, 57-72. doi:10.1007/BF00855426.

[14] Voigt, W., 1966. Lehrbuch der Kristallphysik: Mit Ausschluß der Kristalloptik. Vieweg+Teubner Verlag, Wiesbaden and s.l. doi:10. 1007/978-3-663-15884-4. 